\title{
Cloning, characterization and phylogenetic relationships of stxI, a endoxylanase-encoding gene from Streptomyces thermonitrificans NTU-88
}

\author{
Hsueh-Ling Cheng a , Pei-Min Wang a, Yu-Chi Chen ${ }^{\mathrm{b}}$, \\ Shang-Shyng Yang ${ }^{\text {a,c }}$, Yo-Chia Chen ${ }^{\mathrm{a}, *}$ \\ a Institute of Biotechnology, National Pingtung University of Science and Technology, Pingtung, Taiwan, ROC \\ ${ }^{\mathrm{b}}$ Department of Food Science, National Pingtung University of Science and Technology, Pingtung, Taiwan, ROC \\ ${ }^{\mathrm{c}}$ Department of Biochemical Science and Technology, National Taiwan University, Taipei, Taiwan, ROC
}

Received 28 August 2006; received in revised form 17 November 2006; accepted 20 November 2006

Available online 9 January 2007

\begin{abstract}
A thermostable xylanase gene (stxI) obtained from Streptomyces thermonitrificans NTU-88 on domain analysis revealed an N-terminal catalytic domain featuring homology to a known xylanase within the glycoside hydrolase family 11 . Recombinant STXI retained more than $60 \%$ of its activity following its incubation for at $60^{\circ} \mathrm{C}$ for $24 \mathrm{~h}$. These characteristics were close to thermophile and mesophile Streptomyces strains. The main hydrolysis products of xylan degraded by STXI included large xylooligosaccharide fragments. These results indicated that STXI was a typical endoxylanase. As regards the phylogenetic relationships of GH11, STXI and the other xylanase deriving from Streptomyces were included in a subgroup of the aerobic bacterial group. This result implied that the evolutionary relationships between the various xylanases deriving from Streptomyces strains were convergent.
\end{abstract}

(C) 2006 Elsevier Ltd. All rights reserved.

Keywords: Streptomyces thermonitrificans; Xylanase gene; Phylogenetic analysis; Glycosyl hydrolase family 11

\section{Introduction}

Xylan, the major hemicellulose component of the plant cell wall, is a polymer with a linear backbone of $\beta-1,4-\mathrm{D}-$ xylopyranoside residues, residues which are commonly substituted by acetyl, arabinosyl, and glucuronosyl groups (Collins et al., 2005). Due to the structural complexity of xylan, the synergistic actions of a series of enzymes are necessary to hydrolyze xylan into simple sugars. Amongst the various xylanolytic enzymes, endo- $\beta-1,4-x y l a n a s e(\beta-1,4-\mathrm{D}-$ xylan xylanohydrolase; EC 3.2.1.8) has been termed "true xylanase" for its specificity to xylan degradation (Collins et al., 2005), the enzyme randomly cleaving the $\beta-1,4$

\footnotetext{
${ }^{*}$ Corresponding author. Tel.: +886 8 7703202x5181; fax: +8868 7740550 .

E-mail address: ox@mail.npust.edu.tw (Y.-C. Chen).
}

glycosidic bonds in the xylan backbone, thus bringing about a major reduction in the degree of polymerization of the substrate (Collins et al., 2005).

Endo- $\beta$-1,4-xylanases of glycoside hydrolase family 11 (GH11) are commonly found in fungi, bacteria and actinomyces (Collins et al., 2005). Microorganisms distributed in various environments typically develop various features as regards xylanase so as to acclimatize their growth to the prevailing conditions (Collins et al., 2005; Kulkarni et al., 1999). Mesophilic Streptomyces is one of the major bacteria types found to be growing within normal agricultural-waste composts, and xylanolytic activities are one of the major contributing factors to hemicellulose degradation. During solid-state fermentation, however, the temperature of the bulk of the compost will typically rise from ambient temperature to a temperature range of around $50-70^{\circ} \mathrm{C}$, thus a range of thermostable xylanases are necessary for the activities of mesophilic Streptomyces (Jang and Chen, 2003). 
This feature is also important to many industrial applications such as hemicellulose bioconversion of lignocellulose. In this study, we investigated the biochemical characteristics of the xylanase of Streptomyces thermonitrificans NTU88 by cloning a xylanase gene into Escherichia coli. We also discuss the phylogenetic relationships of GH11 as regards $S$. thermonitrificans NTU-88 and other xylanolytic microorganisms, by analysing certain of their amino-acid sequences.

\section{Methods}

\subsection{Microorganism and DNA extraction}

S. thermonitrificans NTU-88 was grown for five days at $50^{\circ} \mathrm{C}$ in a Mandels-Reese broth (Jang and Chen, 2003). The enriched biomass was collected for the genomic DNA extraction. The protocol for DNA extraction was based upon phenol-chloroform extraction (Chen et al., 2003).

\subsection{Cloning procedures and recombinant xylanase expression}

The PCR reaction adopted herein was used for the amplification of xylanase genes obtained from the genomic DNA samples extracted from $S$. thermonitrificans NTU-88. Two primers, stxF (5'CCAGACCGGCACCCACAACGGC- $3^{\prime}$ ) and stxR (5'GCTGACCGTGGGCCAGGTCC- $3^{\prime}$ ) were used in the PCR reaction. The purified PCR product (stxI gene) was cloned to the pGEM-T-Easy vector and pET21a (Novagen Inc., Germany) for DNA-sequence determination and STXI expression, respectively. The stxI gene-transformed clone was grown in Luria-Bertani broth for for the induction of STXI (Huang et al., 2005). The molecular mass of the recombinant STXI was estimated by means of a slab-gel zymogram as previously described by Theather (Theather and Wood, 1982).

\subsection{Enzyme assays}

The xylanase activity of recombinant STXI was measured by a method as described in 2005 by Huang et al. (2005). For estimation of the reaction temperature and $\mathrm{pH}$ optima of STX, the relative activity of xylanase was determined at different temperature levels as also different $\mathrm{pH}$ values. The relative temperature stability of the tested xylanase was determined by incubating the enzyme, aseptically, in the absence of any substrate, and at various different temperatures $\left(10-100^{\circ} \mathrm{C}\right.$ ) for a period of $4 \mathrm{~h}$ (Sengupta et al., 2000). The influence of a variety of metal ions upon the enzyme's activity was investigated by addition of various different metal salts $\left(\mathrm{Ca}^{2+}, \mathrm{Co}^{2+}, \mathrm{Cu}^{2+}, \mathrm{Fe}^{2+}, \mathrm{Hg}^{2+}\right.$, $\mathrm{Mg}^{2+}, \mathrm{Mn}^{2+}$ and $\mathrm{Ni}^{2+}$ ) at a final metal ion concentration of $10 \mathrm{mM}$ in the reaction mixture (Sengupta et al., 2000). The specificity of STXI was tested for its ability to hydrolyse a variety of presented substrates. Hydrolysis products of xylan were identified by thin-layer chromatography (TLC).

\subsection{Phylogenetic analysis}

The deduced amino-acid sequence of stxI was aligned by use of CLUSTAL X software (Thompson et al., 1997) with available GH11 xylanase sequences retrieved from the CAZy database (Henrissat and Bairoch, 1996). The phylogenetic tree based upon the neighbour-joining (NJ) algorithm was generated by using MEGA software (Kumar et al., 2004). The resultant unrooted tree topologies were evaluated in light of a bootstrap analysis of the neighbourjoining method based upon 1000 resamplings using the seqboot and consense programs in the MEGA package.

\section{Results and discussion}

\subsection{The nucleotide and deduced amino-acid sequences of stxI}

The total length of the stxI was $839 \mathrm{bp}$ and it contained a putative coding region encoding a polypeptide of aminoacids with a molecular mass of $30.4 \mathrm{kDa}$. Analysis of the induced crude extracts by zymogram revealed that the activity of xylanase was remained and featured one clear band with an apparent molecular weight of $32 \mathrm{kDa}$ (Fig. 1). An analysis of stxI revealed that the DNA featured an overall $\mathrm{G}+\mathrm{C}$ content of $64.96 \%$ and a $\mathrm{G}+\mathrm{C}$ content of $92.1 \%$ in the third position of the codons. This nucleotide composition featuring a rather high $\mathrm{G}+\mathrm{C}$ content was typical of many thermophilic microorganisms (Gilkes et al., 1991). According to the sequence-based glycosyl hydrolase classification (Henrissat and Bairoch, 1996), a putative

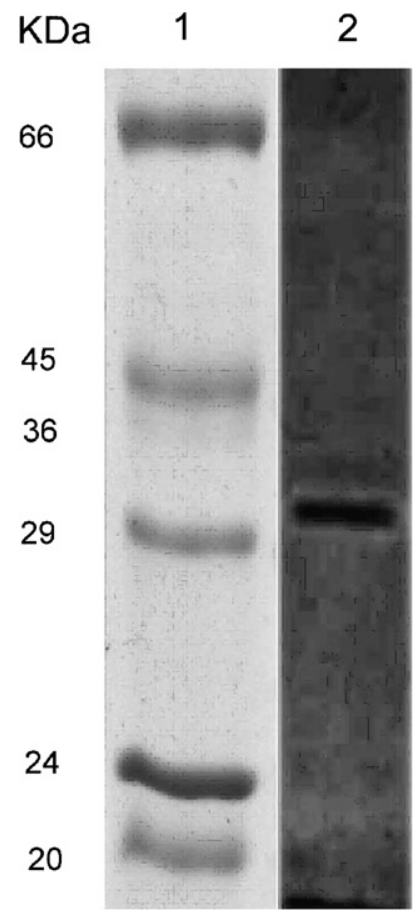

Fig. 1. Zymogram analysis of E. coli lysate using xylan substrate and Congo-red staining. Lane: 1: Protein standards. Lane 2: Cell lysate of E. coli $\mathrm{BL} 21$ (DE3) harboring pET21C-st $x \mathrm{I}$. 


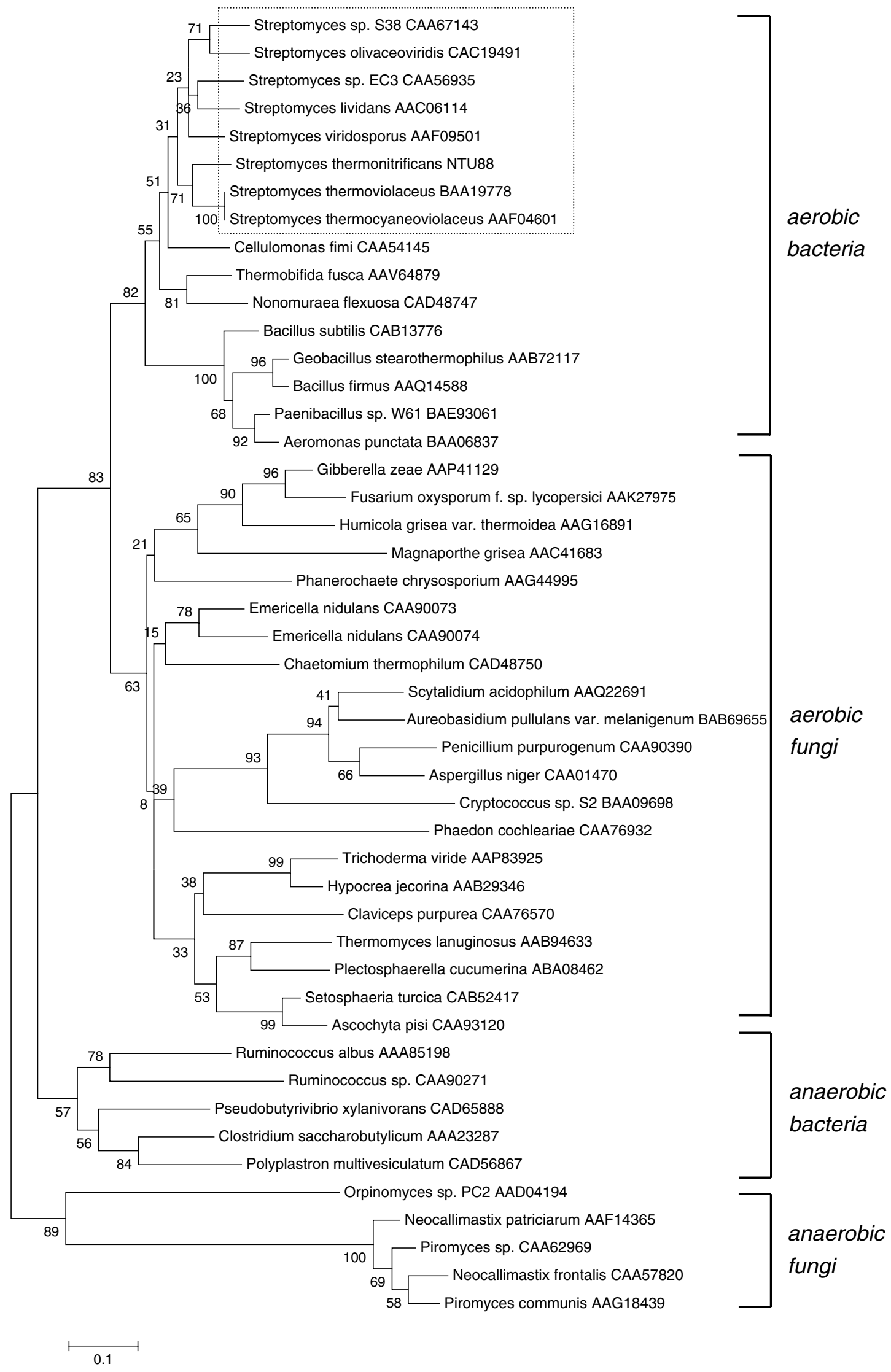

Fig. 2. Neighbour-joining tree based upon partial GH11 xylanase sequences of 47 xylanolytic microbes. The numbers at the nodes indicate the level (\%) of bootstrap support based upon a neighbour-joining analysis of 1000 resampled data sets. The accession numbers of amino-acid sequences are listed at the end of the scientific name of microorganisms. 
conserved domain of GH family 11 was detected at position 106-516 of STXI. Interestingly, an additional cellulose-binding domain (CBD) conserved with CBD family two was found at the C-terminal (position 582-834) of STXI. Some types of CBD are found to be able to stabilize the structure of xylanase. Such modules have also been found to be present within mesophilic bacteria such as Cellulomononas fimi and Bacillus spp. Thus, CBD2 was thought to be capable of thermostabilizing the activity of the xylanase from Streptomyces when in a mesophilic environment (Collins et al., 2005; Gilkes et al., 1991).

\subsection{Enzyme characteristics of STXI}

STXI remained active over a very broad $\mathrm{pH}$ range of 5-9 and xylanase was active between 30 and $60^{\circ} \mathrm{C}$ with an optimum at around $50^{\circ} \mathrm{C}$. The enzyme showed rather good stability from 10 to $50^{\circ} \mathrm{C}$, whereas above $60^{\circ} \mathrm{C}$ the enzyme's stability decreased rapidly. The thermostability of the enzyme appeared to be quite pronounced within the mesophilic range. The biochemical characteristics of the recombinant STXI are similar to those reported for many of the enzymes of Streptomyces strains isolated from compost (Ninawe and Kuhad, 2005, 2006; Ruiz-Arribas et al., 1998). Various metal ions examined revealed either an enhancing or inhibiting effects were insignificant except $\mathrm{Hg}^{2+}$. These results suggested that the xylanases were not a metalloprotein (Heck et al., 2006).

STXI appeared to be specific for xylan degradation, the highest activity being found on oat-spelt xylan; however, the enzyme also displayed diminished activity toward carboxylmethyl cellulose, avicel and $\beta$-glucan as compared to xylan substrates. The hydrolysis products released from oatspelt xylan by STXI consisted of a mixture of large xylooligosaccharide fragments, whereas xylotriose, xylobiose and xylose were not visualized in the TLC plate. This indicated that the mode of action of xylanase was of an endo-type hydrolysis and this type of xylanase could be applied in the prebleaching of eucalyptus kraft pulp (Dhillon et al., 2000).

\subsection{Phylogenetic relationships}

A relatedness tree was constructed for $\mathrm{GH} 11$, the tree including the sequence of the catalytic region of STXI in addition to the sequences of the sequences of GH11 deriving from various microorganisms. The analysis of NJ revealed the division of the homology to GH11 xylanase into four groups, including aerobic bacteria, aerobic fungi, anaerobic bacteria and anaerobic fungi (Fig. 2). The GH11 xylanases of Streptomyces were clustered in the subgroup of aerobic bacterium group. The sequence homology of these different xylanases appeared to be consistent with a hypothesis that the xylanases of GH11 evolved together within the Streptomyces that contained them.

In 1991, Glikes et al. reported that the $\beta$-1,4-glycanases of various microorganisms arose from limited progenitor sequences by mutation, domain fusion and shuffling.
These changes could give critical insights into the ways in which the enzymes interact with certain substrates, or how these enzymes "fit into" their environments. For example, non-catalytic thermostabilizing domains have been found to be present in xylanases of thermophilic bacteria (Gilkes et al., 1991), whereas the conserved triad (Val-Val-Asn or Val-Val-Asp) has been identified as being present only in the xylanases from alkaliphilic organisms (Kulkarni et al., 1999). In this study, the phylogenetic relationships of GH11 (Fig. 2) suggested that some elements of the catalytic region within GH11 xylanases could vary according to the oxygen requirement of xylanolytic microorganisms.

\section{Acknowledgements}

The financial support of the National Science Council of Taiwan (Grant No. NSC 94-2313-B-020 -018) for this research is greatly appreciated.

\section{References}

Chen, Y.C., Hseu, R.S., Cheng, K.J., 2003. The genetic similarity of different generations of Neocallimastix frontalis SK. FEMS Microbiol. Lett. 221, 227-231.

Collins, T., Gerday, C., Feller, G., 2005. Xylanases, xylanase families and extremophilic xylanases. FEMS Microbiol. Rev. 29, 3-23.

Dhillon, A., Gupta, J.K., Jauhari, B.M., Khanna, S., 2000. A cellulase-poor, thermostable, alkalitolerant xylanase produced by Bacillus circulans AB 16 grown on rice straw and its application in biobleaching of eucalyptus pulp. Bioresour. Technol. 73, 273-277.

Gilkes, N.R., Henrissat, B., Kilburn, D.G., Miller Jr., R.C., Warren, R.A., 1991. Domains in microbial beta-1, 4-glycanases: sequence conservation, function, and enzyme families. Microbiol. Rev. 55, 303-315.

Heck, J.X., Flores, S.H., Hertz, P.F., Ayub, M.A., 2006. Statistical optimization of thermo-tolerant xylanase activity from Amazon isolated Bacillus circulans on solid-state cultivation. Bioresour. Technol. 97, 1902-1906.

Henrissat, B., Bairoch, A., 1996. Updating the sequence-based classification of glycosyl hydrolases. Biochem. J. 316, 695-696.

Huang, Y.H., Huang, C.T., Hseu, R.S., 2005. Effects of dockerin domains on Neocallimastix frontalis xylanases. FEMS Microbiol. Lett. 243, 455-460.

Jang, H.D., Chen, K.S., 2003. Production and characterization of thermostable cellulases from Streptomyces transformant T3-1. World J. Microbiol. Biotechnol. 19, 263-268.

Kulkarni, N., Lakshmikumaran, M., Rao, M., 1999. Xylanase II from an alkaliphilic thermophilic Bacillus with a distinctly different structure from other xylanases: evolutionary relationship to alkaliphilic xylanases. Biochem. Biophys. Res. Commun. 263, 640-645.

Kumar, S., Tamura, K., Nei, M., 2004. MEGA3: Integrated software for molecular evolutionary genetics analysis and sequence alignment. Brief Bioinform. 5, 150-163.

Ninawe, S., Kuhad, R.C., 2005. Use of xylan-rich cost effective agro-residues in the production of xylanase by Streptomyces cyaneus SN32. J. Appl. Microbiol. 99, 1141-1148.

Ninawe, S., Kuhad, R.C., 2006. Bleaching of wheat straw-rich soda pulp with xylanase from a thermoalkalophilic Streptomyces cyaneus SN32. Bioresour. Technol. 97, 2291-2295.

Ruiz-Arribas, A., Zhadan, G.G., Kutyshenko, V.P., Santamaria, R.I., Cortijo, M., Villar, E., Fernandez-Abalos, J.M., Calvete, J.J., Shnyrov, V.L., 1998. Thermodynamic stability of two variants of xylanase (Xys1) from Streptomyces halstedii JM8. Eur. J. Biochem. 253, 462468. 
Sengupta, S., Jana, M.L., Sengupta, D., Naskar, A.K., 2000. A note on the estimation of microbial glycosidase activities by dinitrosalicylic acid reagent. Appl. Microbiol. Biotechnol. 53, 732-735.

Theather, R.M., Wood, P.J., 1982. Use of Congo red-polysaccharide interactions in enumeration and characterization of cellulolytic bacteria from the bovine rumen. Appl. Environ. Microbiol. 37, 777-780.
Thompson, J.D., Gibson, T.J., Plewniak, F., Jeanmougin, F., Higgins, D.G., 1997. The CLUSTAL_X windows interface: flexible strategies for multiple sequence alignment aided by quality analysis tools. Nucleic Acids Res. 25, 4876-4882. 